\title{
Postcumulus processes in layered intrusions
}

\author{
R. S. J. SPARKS, ${ }^{*}$ H. E. HUPPERT, $\dagger$ R. C. KERR, $†$ D. P. McKENZIE*, \& S. R. TAIT* \\ * Department of Earth Sciences, University of Cambridge, Cambridge CB2 3EQ, U.K. \\ $†$ Department of Applied Mathematics and Theoretical Physics, University of Cambridge, Cambridge CB3 9EW, U.K.
}

(Received 30 November 1984; accepted 18 March 1985)

\begin{abstract}
During the postcumulus stage of solidification in layered intrusions, fluid dynamic phenomena play an important role in developing the textural and chemical characteristics of the cumulate rocks. One mechanism of adcumulus growth involves crystallization at the top of the cumulate pile where crystals are in direct contact with the magma reservoir. Convection in the chamber can enable adcumulus growth to occur to form a completely solid contact between cumulate and magma. Another important process may involve compositional convection in which light differentiated melt released by intercumulus crystallization is continually replaced by denser melt from the overlying magma reservoir. This process favours adcumulus growth and can allow adcumulus growth within the pore space of the cumulate pile. Calculations indicate that this process could reduce residual porosities to a few percent in large layered intrusions, but could not form pure monomineralic rocks. Intercumulus melt may also be replaced by more primitive melt during episodes of magma chamber replenishment. Dense magma, emplaced over a cumulate pile containing lower density differentiated melt may sink several metres into the underlying pile in the form of fingers. Reactions between melt and matrix may lead to changes in mineral compositions, mineral textures and whole rock isotope compositions. Another important mechanism for forming adcumulate rocks is compaction, in which the imbalance of the hydrostatic and lithostatic pressures in the cumulate pile causes the crystalline matrix to deform and intercumulus melt to be expelled. For cumulate layers from 10 to 1000 metres in thickness, compaction can reduce porosities to very low values $(<1 \%)$ and form monomineralic rocks. The characteristic time-scale for such compaction is theoretically short compared to the time required to solidify a large layered intrusion. During compaction changes of mineral compositions and texture may occur as moving melts interact with the surrounding matrix. Both compaction and compositional convection can be interrupted by solidification in the pore spaces. Compositional convection will only occur if the Rayleigh number is larger than 40 , if the residual melt becomes lower in density, and the convective velocity exceeds the solidification velocity (measured by the rate of crystal accumulation in the chamber). Orthocumulates are thus more likely to form in rapidly cooled intrusions where residual melt is frozen into the pore spaces before it can be expelled by compaction or replaced by convection.
\end{abstract}

\section{Introduction}

In recent years the physical ideas embodied in cumulus theory have been re-examined. Detailed studies of a number of major layered intrusions have recognized field relationships that appear inconsistent with a crystal settling model (Campbell, 1978; Morse, 1979; McBirney \& Noyes, 1979; Irvine, Keith \& Todd, 1983; Wilson \& Larsen, 1985). There are good arguments in favour of an in situ crystallization mechanism, although textural evidence for crystal settling is still compelling in some rocks, such as the Rhum peridotites (Brown, 1956; Tait, 1985). Despite the uncertainties that currently exist on the origin of these rocks, the cumulus classification survives. This is because Wager \& Deer (1939), Wager, Brown \& Wadsworth (1960) and Jackson (1961) recognized that many of the rocks from layered intrusions consist of an initial crystal framework with interstitial pore material. Those rocks with a substantial proportion of pore material can be termed orthocumulates. Those rocks with little or no surviving pore material can be termed adcumulates and those with intermediate amounts can be called mesocumulates. Thus the cumulate classification is appropriate irrespective of the mode of formation (Irvine, 1982).

Postcumulus processes, which occur after the development of the initial cumulus framework (Wadsworth, 1985), can involve either complete solidification or recrystallization under subsolidus conditions. Several ideas on postcumulus processes have recently emerged, partly based on experimental investigations and theoretical advances in the fluid dynamical aspects of crystallization, porous media convection and compaction of partially molten rock. Much of the data and detailed analyses on these ideas are contained in Kerr \& Tait $(1985 a, b)$, in the unpublished Ph.D. theses of R. C. Kerr and S. R. Tait (University of Cambridge, 1984) and in recent work on compaction (McKenzie, 1984, 1985; Richter \& McKenzie, 1984). Important concepts have come from Morse $(1969,1979,1982)$ and Irvine (1980), based on studying the petrography and chemistry of the rocks. Recent investigations of layered intrusions, in particular on Rhum, have suggested that postcumulus processes are more diverse and complicated than 
originally envisaged in the classic cumulus theory (for example Young, 1984; Butcher, Young \& Faithfull, 1985; Tait, 1985; Young \& Donaldson, 1985). This paper provides a synopsis of these new ideas and concentrates on fluid dynamical phenomena that are likely to be associated with consolidation of the pore spaces and compaction of the matrix.

Although this paper is not specifically concerned with Rhum, the authors felt that it is appropriate to include a review of postcumulus processes in this special issue. Rhum has had a central role in developing cumulus theory (Brown, 1956; Wager, Brown \& Wadsworth, 1960). Several papers in this volume illustrate that Rhum continues to inspire imaginative new research into the origin of layered rocks. In particular the issue highlights the importance of intercumulus melt circulation which has also been emphasized in the experimental and theoretical studies reported here.

\section{Cumulus theory}

The theory of Wager, Brown \& Wadsworth (1960) was based on the concept of crystal settling and ideas concerning postcumulus processes originally conceived by Hess $(1939,1960)$. At the orthocumulate extreme they envisaged rapid accumulation of crystals which trapped interstitial liquid. During subsequent equilibrium crystallization the trapped pore liquid could react continuously with the cumulus crystals to form lower temperature solid solutions. Some cumulus minerals, notably plagioclase, are slow to equilibrate and may become chemically zoned. Eventually new phases nucleate and grow in the pore space. The adcumulate extreme was thought to occur under conditions of slow crystal accumulation. The cumulus crystals were thus able to maintain chemical communication with the main magma reservoir by diffusion of components through the interstitial melt, and thus could grow at constant composition until virtually all the pore space was eradicated. Wager, Brown \& Wadsworth (1960) recognized the difficulty of static diffusion being a viable mechanism deep in the cumulate pile and argued that adcumulus growth would be most effective in the uppermost layer of the cumulate pile. These ideas assumed that compaction was unimportant (Wadsworth, this volume) and that the consolidation of cumulate piles was essentially a cementation process.

As recognized by Morse $(1979,1982)$, the distinction between cumulus crystals and pore material can be retained without implying a sedimentary origin for the cumulus framework. If the rate of in situ growth is high, then crystals may form a relatively open framework containing trapped melt. On the other hand if the solidification rate is low then there could be time for adcumulus growth by chemical diffusion to occur at the topmost layer of cumulus grains in direct contact with the magma. In the extreme case, the distinction between cumulus crystal and pore material loses meaning since the model does not necessarily involve the formation of a network of pores surrounded by a crystal framework. By the envisaged process, a sharp, well-defined interface develops between totally solid adcumulate rock and the magma. However, as Wadsworth (this issue) has pointed out, there is evidence that adcumulus growth can occur within cumulate piles, so a model of adcumulus growth at the interface between the magma and the floor may not be the only mechanism operating in nature. A model of adcumulus growth by static diffusion between pores deep within the cumulus pile and the chamber is physically implausible (Wager, Brown \& Wadsworth, 1960; Hess, 1972; Tait, Huppert \& Sparks, 1984). Thus, alternative mechanisms to that involving adcumulus growth at the magma-solid interface are required.

Workers on layered intrusions have long recognized the important role of convection (Wager \& Deer, 1939; Morse, 1969). Recent developments in fluid dynamics have, however, recognized a number of new physical phenomena, which may have an important influence on the evolution of layered igneous rocks. Crystallization from a saturated solution often involves vigorous convective motions which can strongly influence how solidification occurs (Chen \& Turner, 1980; Huppert \& Sparks, 1984). These effects could influence events both at the interface of the cumulus pile with the magma and within the pore space deep within the pile. In addition, Irvine (1980) has recognized geological features which suggest that compaction is an important process. McKenzie (1984, 1985) and Richter \& McKenzie (1984) demonstrated that in a partially molten system the crystalline matrix can deform, resulting in two-phase flow, extraction of pore liquid and compaction of the matrix. These latter theoretical studies confirm that compaction is much more effective than was formally thought and should be an important factor in the formation of layered intrusions. In the following sections we assess how fluid dynamical ideas are likely to influence the interpretation of cumulate rocks.

\section{Compositional convection}

When a crystal grows from a liquid a thin film or boundary layer of melt is formed adjacent to the crystal faces. This film is depleted in chemical components taken into the crystalline solid and consequently there is a compositional and hence a density gradient across the film. Under suitable conditions the film will be dynamically unstable and can convect away. Several studies have argued that such compositional convection will be of major importance in magma chambers (Morse, 1969; Chen \& Turner, 1980; McBirney, 1980; Huppert \& Sparks, 
1984). The relevance of this process for cumulate rocks is that convection is a much more effective way than static diffusion of maintaining a constant melt composition adjacent to a growing crystal face. Compositional convection and perhaps thermal convection may consequently be major factors in enabling adcumulus growth to occur.

\section{3.a. Dynamical principles}

During crystallization from a basaltic melt changes in density are usually dominated by compositional effects (Morse, 1969; Sparks \& Huppert, 1984). In crystallization of olivine and pyroxene, for example, residual melts become lower in density despite the decrease in temperature. When plagioclase is a major crystallizing phase, the residual melt often becomes denser. Such compositional convection is generally dominant over motions that might be induced by purely thermal effects.

In the case of crystallization on the floor of a magma chamber or within the underlying cumulate pile, experimental and theoretical studies (reviewed by Huppert \& Sparks, 1984) suggest a limited number of arrangements. In one situation crystallization can release heavy liquid which must sink or simply remain adjacent to the crystal if the underlying material contains even heavier liquid or is impermeable. In other situations, where compositional effects dominate, crystallization can lead to the release of light residual fluid at or beneath the chamber floor which can convect away to be replaced by heavier liquid from the chamber. Figure 1 shows an experiment in which an aqueous solution of $\mathrm{CuSO}_{4}$ and $\mathrm{Na}_{2} \mathrm{SO}_{4}$ is being cooled from below. Crystals of $\mathrm{CuSO}_{4} \cdot 5 \mathrm{H}_{2} \mathrm{O}$ and $\mathrm{Na}_{2} \mathrm{SO}_{4} \cdot 10 \mathrm{H}_{2} \mathrm{O}$ are growing within a basal layer of glass balls underlying a large reservoir of the solution. As the crystals grow, thin streams of solute-depleted fluid emerge from the porous medium and rise to mix with the overlying reservoir. Continuity requires that an equal flux of solute-enriched solution descends into the porous media. Adcumulus growth of $\mathrm{CuSO}_{4} \cdot 5 \mathrm{H}_{2} \mathrm{O}$ and $\mathrm{Na}_{2} \mathrm{SO}_{4} \cdot 10 \mathrm{H}_{2} \mathrm{O}$ crystals is taking place between the glass balls.

Several fundamental points concerning compositional convection should be noted:

(i) Vigorous compositional convection can occur although the system is thermally stable, as illustrated in the experiment where the fluid is being cooled from below. The compositional effects on solution density overwhelm the stabilizing temperature gradient.

(ii) The convective patterns can sometimes be quite different in compositional convection to those observed during thermal convection. For example, in thermal

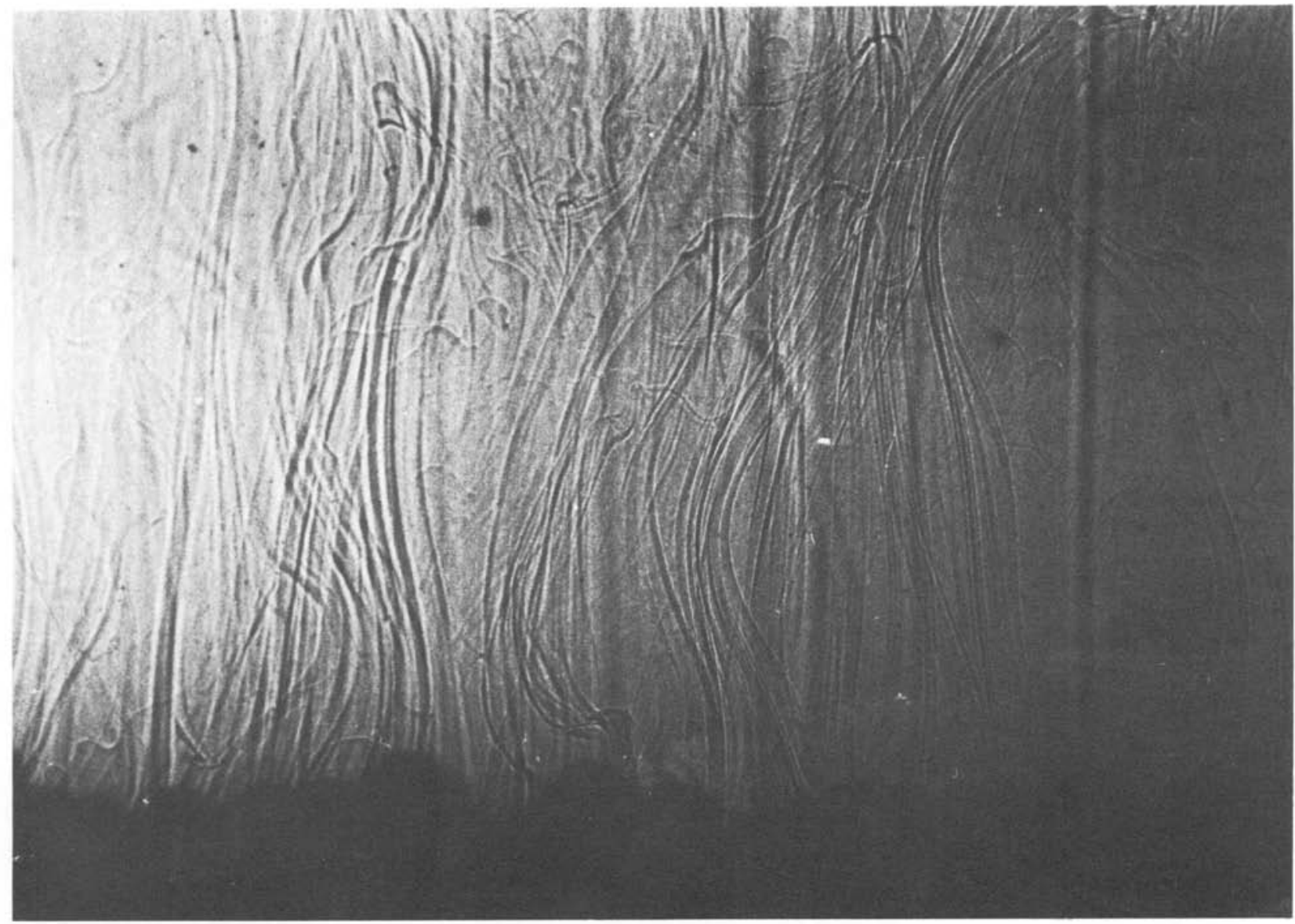

Figure 1. Streams of light residual fluid are rising from a bed of glass balls $(0.5 \mathrm{~cm}$ diameter $)$ in which crystallization of $\mathrm{CuSO}_{4} .5 \mathrm{H}_{2} \mathrm{O}$ and $\mathrm{Na}_{2} \mathrm{SO}_{4} \cdot 10 \mathrm{H}_{2} \mathrm{O}$ is taking place. The photograph shows a width of $5 \mathrm{~cm}$. 
convection caused by uniform heating of a surface, motions can be quite chaotic at high Rayleigh numbers with rapid horizontal mixing. In compositional convection each crystal provides the source of a very thin uprising plume or stream. The motion is dominantly vertical with limited horizontal mixing due to the much lower chemical diffusivity in comparison to thermal diffusivity.

(iii) The appropriate boundary layer thicknesses are proportional to the relevant coefficient of diffusion and are hence much less for compositional convection than for thermal convection. Compositional boundary layers next to growing crystal faces can be as little as tens or hundreds of microns. This latter type of convection can therefore more readily operate in small pores.

(iv) The experiments show that adcumulus growth can occur within a porous medium and need not be confined to the uppermost layers of a cumulate pile.

\section{3.b. Interface between the magma and cumulate pile}

The idea that adcumulus growth can occur directly on the floor of the chamber is supported by experimental studies. We have carried out a number of experiments in which an aqueous solution with a non-eutectic composition is cooled from either the roof or floor of the container. In some experiments a solid 'adcumulate' region made of interlocking crystals forms with a sharp interface against the overlying liquid.

Figure 2 shows a case where $\mathrm{CuSO}_{4} \cdot 5 \mathrm{H}_{2} \mathrm{O}$ and $\mathrm{Na}_{2} \mathrm{SO}_{4} \cdot 10 \mathrm{H}_{2} \mathrm{O}$ crystals grew to form an impermeable layer on the surface of a bed of glass balls. The system was being cooled from below, and crystals nucleated throughout the porous medium and on its upper surface. However, the permeability of the porous medium was sufficiently low that compositional convection was weak within the pore space. Light, residual fluid released from crystals that nucleated on the upper surface could convect away much more rapidly than the fluid released from crystals within the porous medium. Consequently, the crystals on the upper surface continued to grow as they were supplied with solute-rich solution by vigorous compositional convection that took place above. The $\mathrm{CuSO}_{4}-\mathrm{Na}_{2} \mathrm{SO}_{4}$ layer contained no ice and is effectively a pure adcumulate. Thus, in some circumstances, adcumulus crystal growth can be more effective on the surface than in the interior of the porous medium.

We have conducted experiments with a wide variety of aqueous systems in which cooling and crystallization occurs at the floor of a tank containing an homogeneous fluid. Some of these experiments have been described by Kerr \& Turner (1982) and Huppert

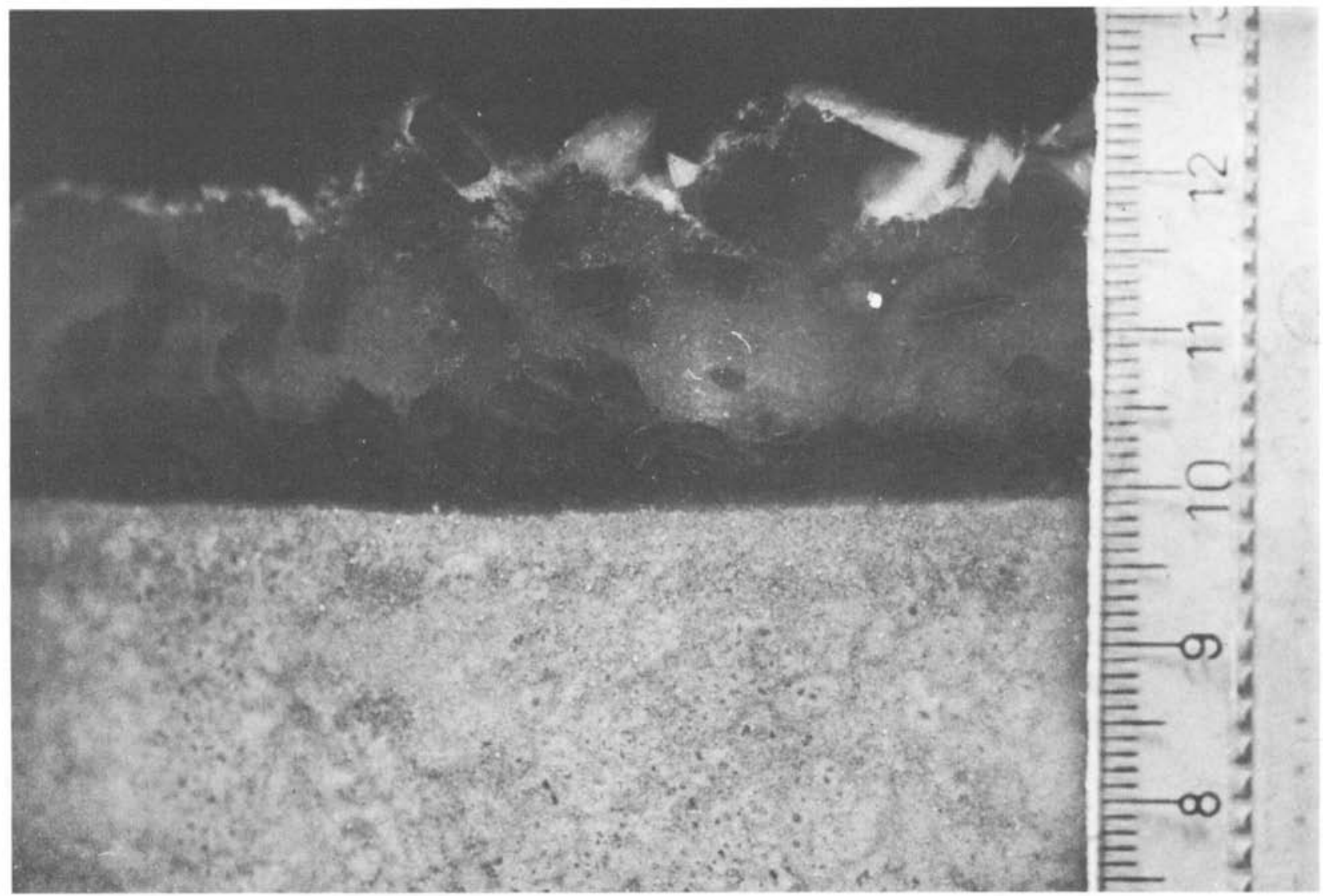

Figure 2. The photograph shows an impermeable layer of $\mathrm{CuSO}_{4} \cdot 5 \mathrm{H}_{2} \mathrm{O}$ crystals (dark) and $\mathrm{Na}_{2} \mathrm{SO}_{4} \cdot 10 \mathrm{H}_{2} \mathrm{O}$ crystals overlying a porous medium of $0.032 \mathrm{~cm}$ glass balls. The speckled appearance of the glass balls is due to many tiny crystals of $\mathrm{CuSO}_{4} .5 \mathrm{H}_{2} \mathrm{O}$ and $\mathrm{Na}_{2} \mathrm{SO}_{4} \cdot 10 \mathrm{H}_{2} \mathrm{O}$ which grew in the pores. In this experiment convection was weak (initial Rayleigh number $10^{3}$ ). The scale on the right is shown in centimetres. 
\& Worster (1985). For most systems in which light, water-enriched fluid is released by crystallization of the particular salt compound, a solid layer of hydrated salt is formed with little or no ice. However, in experiments on the same systems carried out on the ice side of the eutectic, residual fluid denser than the original is released. In this case the dense fluid fills crevices and interstices between the ice crystals and the solid product contains crystals of the salt as well as ice. In geological parlance these materials would have a more orthocumulate character. The experimental studies suggest that the density changes caused by crystallization on the surface of a chamber floor should have an important influence on whether adcumulate 'hardgrounds' can form in magma chambers.

Another factor, that cannot yet be investigated in the tank experiments, is the influence of thermal convection in a magma chamber. Vigorous thermal convection could sweep away residual magma of greater density from the floor and replenish the environment of crystal growth with fresh magma to promote adcumulus growth. Morse (1969) invokes this process in the formation of anorthosite layers in the Kiglapait intrusion.

In summary, the concept that adcumulates may form as a virtually solid 'hardground' on the base of a magma chamber is plausible. As argued by Wager, Brown \& Wadsworth (1960) and Morse $(1979,1982)$, this process should be most effective when the accumulation or solidification rate is very slow. Experimental studies confirm that this mechanism will be more effective if aided by compositional convection.

\section{3.c. The interior of the cumulate pile}

Slumping of layers to depths of many tens of centimetres or metres (Jackson, 1961; Wadsworth, 1985) and the occurrence of orthocumulates and mesocumulates with clear evidence of trapped melt (Wager \& Brown, 1968) suggest that substantial depths of porous material commonly form in magma chambers. Further, geochemical evidence for "infiltration metasomatism ' (Irvine, 1980) and other data presented by various authors (Butcher 1985; Butcher, Young \& Faithfull, 1985; Palacz \& Tait, 1985; Tait, 1985; Young \& Donaldson, 1985) imply that circulation of intercumulus melt can occur within cumulate piles. There is also evidence that adcumulus growth occurs within crystal piles. In the case of Rhum, the peridotites are considered to represent rapid accumulations of olivine crystals following replenishment (Brown, 1956; Tait, 1985). The layers of loosely packed olivine crystals had perhaps $35 \%$ initial porosity and can be tens of metres thick. As argued by Wadsworth (1985) and others, the pore space ultimately was filled by an assemblage of plagioclase and clinopyroxene. The composition of the interstitial material in the Rhum peridotite does not correspond to a sensible liquid and is partly adcumulate in character. Thus, the 'hardground' model (outlined in Section 3.b) cannot be the only mechanism of forming adcumulates.

Tait, Huppert \& Sparks (1984) proposed that intercumulus melt convection during pore-space crystallization could be a mechanism of adcumulus growth at some depth within cumulate piles. This possibility was also noted by Irvine (1980). To test this hypothesis further, Kerr \& Tait (1985a) carried out a series of experiments on crystal growth in a porous medium using the $\mathrm{Na}_{2} \mathrm{SO}_{4}-\mathrm{CuSO}_{4}-\mathrm{H}_{2} \mathrm{O}$ ternary system. In the experiments a layer of glass balls or $\mathrm{CuSO}_{4}$ crystals (10 to $14 \mathrm{~cm}$ deep) was placed on the floor of a circular perspex cylinder, $29 \mathrm{~cm}$ in diameter and $60 \mathrm{~cm}$ high. The tank was then filled with a solution nearly saturated in both $\mathrm{Na}_{2} \mathrm{SO}_{4}$ and $\mathrm{CuSO}_{4}$ and then cooled from below by passing cold methylated spirits though a basal brass plate. The experiments involved systematic variations in starting compositions, cooling rates and grain size and therefore permeability of the porous media. The detailed results are described by Kerr \& Tait (1985a) and here we only summarize the main observations and conclusions.

Each experiment began with a porosity of about $35 \%$. As cooling proceeded the solutions soon reached saturation and crystals of either $\mathrm{CuSO}_{4} \cdot 5 \mathrm{H}_{2} \mathrm{O}$, $\mathrm{Na}_{2} \mathrm{SO}_{4} \cdot 10 \mathrm{H}_{2} \mathrm{O}$ or both nucleated within the pore spaces. For those experiments with high permeability (large glass balls or crystals) and slower cooling rates, streams of light residual fluid were observed to emerge from the upper surface of the porous media as illustrated in Figure 1. Eventually liquid trapped in the pore space reached the ternary eutectic temperature, and ice as well as $\mathrm{CuSO}_{4} \cdot 5 \mathrm{H}_{2} \mathrm{O}$ and $\mathrm{Na}_{2} \mathrm{SO}_{4} \cdot 10 \mathrm{H}_{2} \mathrm{O}$ formed a well-defined solid layer at the base of the tank (Fig. $3 a$ ). This entirely solid layer grew with time and moved up through the porous medium until there was an entirely solid, lower layer consisting of glass balls and interstitial crystalline solid. The composition and temperature of the overlying fluid was monitored throughout each experiment and the solid product was also analysed after each run. Estimates of the convective velocities of plumes emerging from the porous medium were also made.

The main results of these studies are summarized below:

(i) In those experiments where vigorous compositional convection was observed to come from the porous medium, the compositions of the final interstitial solid was enriched in $\mathrm{CuSO}_{4}$ and $\mathrm{Na}_{2} \mathrm{SO}_{4}$ in comparison to the initial homogeneous solution. Adcumulus growth had occurred and the process was only halted when ice nucleated in the pore space to form the eutectic solid layer.

(ii) The experiments with large balls (and therefore 

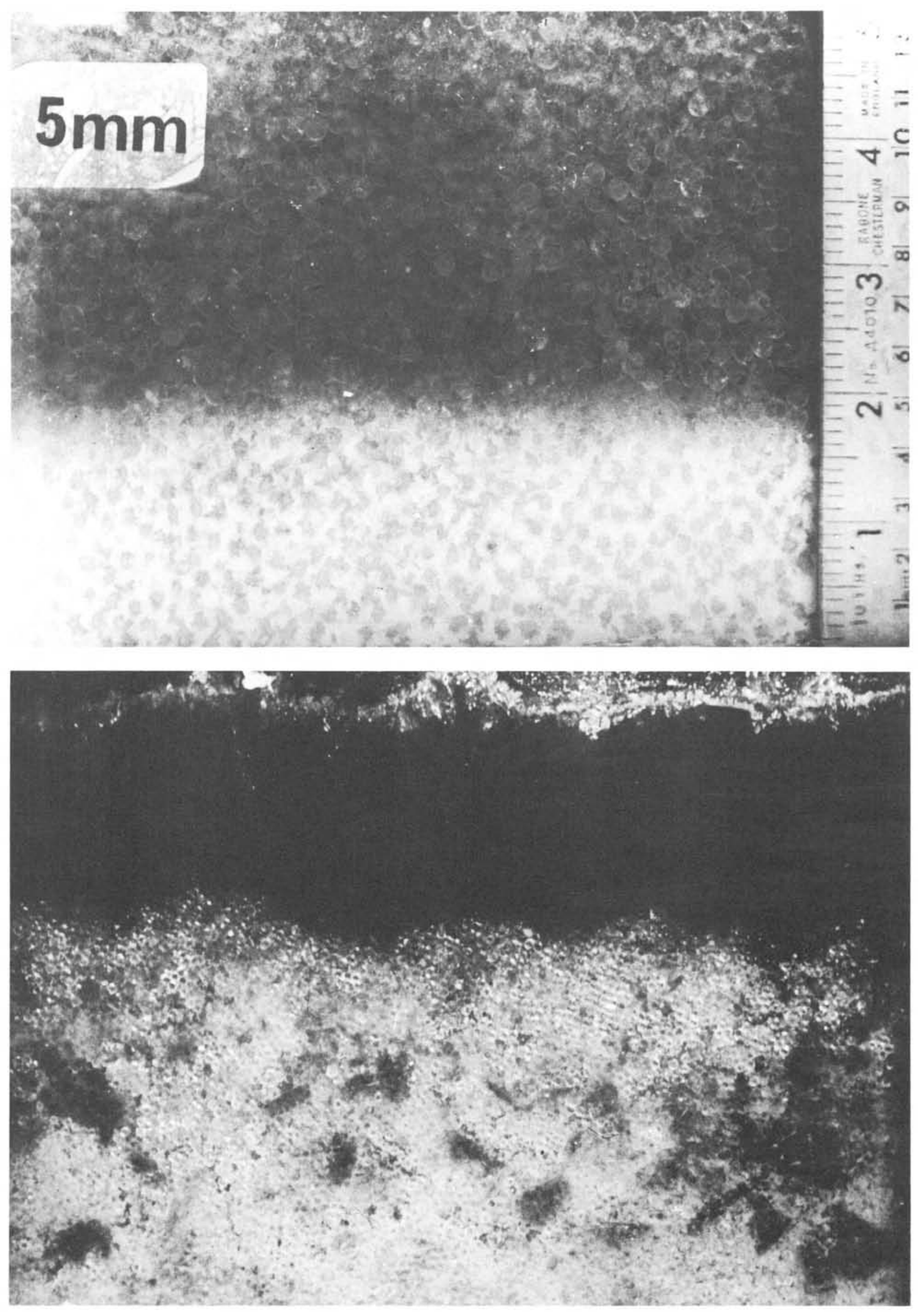

Figure 3. (a) The photograph shows the interior of a porous medium of $5 \mathrm{~mm}$ glass balls in which crystals of $\mathrm{CuSO}_{4} .5 \mathrm{H}_{2} \mathrm{O}$ and $\mathrm{Na}_{2} \mathrm{SO}_{4} \cdot 10 \mathrm{H}_{2} \mathrm{O}$ have grown. In the lower $25 \mathrm{~cm}$ of the tank a layer of totally solid ice and hydrated salt crystals has formed with a sharp boundary with overlying ice-free porous medium. (b) The photograph shows dark poikilitic crystals of $\mathrm{CuSO}_{4} \cdot 5 \mathrm{H}_{2} \mathrm{O}$ which have grown in a porous medium $(d=0.032 \mathrm{~cm})$ and have enclosed several glass balls. The upper black layer is a capping layer as described in Figure 2. 
higher initial permeability) showed the most vigorous circulation and the greatest enrichment of the pore material in $\mathrm{CuSO}_{4}$ and $\mathrm{Na}_{2} \mathrm{SO}_{4}$.

(iii) In an experiment with the smallest balls (diameter $0.32 \mathrm{~mm}$ ) and lowest permeability, no convection was observed and the pore material had a composition indistinguishable from the initial solution.

(iv) In those experiments with only weak convection an impermeable capping layer formed as shown in Figure 2.

(v) Textural features included development of 'poikilitic' intercumulus $\mathrm{CuSO}_{4} \cdot 5 \mathrm{H}_{2} \mathrm{O}$ crystals (Fig. 3b).

In order to apply these results and observations to magma chambers a theoretical treatment of the convection and crystallization in porous media is now developed. The occurrence of significant adcumulus growth by compositional convection requires that several physical conditions are met. The first requirement is that crystallization produces lower density residual melt so that compositional convection can take place on the floor. If denser residual fluid is produced convection could still occur if there is a fresh supply of lower density fluid from beneath or even denser fluid from above. However, for chambers as they are conventionally perceived adcumulus growth within the cumulus pile is much more probable if light residual liquid is produced. Since the fractional crystallization of plagioclase-rich cumulate assemblages is thought to generate dense residual melts (Sparks \& Huppert, 1984), we anticipate that compositional convection will be most effective in mafic cumulates, where melt density decreases with fractionation.

A second requirement is that the solutal Rayleigh number, $R a_{s}$, exceeds a critical value of about 40 (Lapwood, 1948). This parameter can be defined as

$$
R a_{s}=\frac{k g h \Delta \rho}{\mu D},
$$

where $k$ is the permeability, $g$ is the acceleration due to gravity, $h$ is the depth of the porous medium, $\mu$ is the fluid viscosity, $D$ is the effective chemical diffusivity in the fluid and $\Delta \rho$ is the density difference in the liquid driving the convection. Table 1 compares the ranges of physical properties and parameters encountered in the experiments and estimates for magma chambers. For cumulate piles with initial porosities of 0.3 to 0.5 and depths of $1-100 \mathrm{~m}$ the solutal Rayleigh numbers range from $10^{3}$ to $10^{7}$, suggesting that compositional convection should be vigorous in magma chambers where low density residual liquids are being generated.

$A$ third requirement concerns the relative rates of convection and solidification. As crystals fill the pore space, permeability decreases, and therefore the convection velocity should be reduced. Eventually the residual porosity should become sufficiently small that the pore fluid will freeze in situ before circulation could take place. In this situation the relative magnitudes of the solidification velocity $\left(v_{\mathrm{s}}\right)$ and the convective velocities $\left(v_{c}\right)$ become important. In this context we take solidification velocity to be the velocity at which the solid boundaries at the chamber margins move inwards and is approximately the same as the accumulation rate (Irvine, 1970). The convection velocity refers to the velocity of melt within the pore space. Figure 4 plots the initial and final porosities against ball diameter in four of the experiments. Residual porosities were estimated by comparing the final solid compositions to the average solution compositions. A family of parallel curves indicates the approximate value of the ratio of convection velocity to the solidification velocity. The hypothesis that we wished to test was that when $v_{\mathrm{c}} / v_{\mathrm{s}}=1.0$ there would be little further adcumulus growth because the pore fluid is frozen in before significant circulation could take place. Figure 4 shows that in the experiments the residual trapped porosity lies between the lines of $v_{\mathrm{c}} / v_{\mathrm{s}}=1$ and 10 when adcumulus growth effectively ceased.

The characteristic convective velocities can be estimated (Kerr \& Tait, 1985a) by the following expression

$$
v_{\mathrm{c}}=\frac{d^{2} g \Delta \rho \epsilon^{4.5}}{10 \mu},
$$

where $\epsilon$ is the porosity, $d$ is the grain diameter and $\Delta \rho$ is the difference between the densities of residual liquid and the original magma. Figure 5 shows the variation of convection velocity against magma viscosity for $d=0.2 \mathrm{~cm}, \Delta \rho=10^{-2} \mathrm{gm} / \mathrm{cm}^{3}$ and for cumulate piles with porosities from 0.05 to 0.4 . As demonstrated in the experiments adcumulus growth is possible if the

Table 1. A comparison of the main parameters affecting compositional convection in the experimental system and in mafic and ultramafic cumulates

\begin{tabular}{lcc}
\hline Parameter & $\begin{array}{c}\text { Experimental } \\
\text { values }\end{array}$ & $\begin{array}{c}\text { Magma chamber } \\
\text { values }\end{array}$ \\
\hline Particle diameter $d(\mathrm{~m})$ & $3 \times 10^{-4}-5 \times 10^{-2}$ & $5 \times 10^{-4}-5 \times 10^{-2}$ \\
Initial porosity & $0.35-0.41$ & 0.3 \\
Permeability $k\left(\mathrm{~m}^{2}\right)$ & $10^{-8}-10^{-10}$ & $10^{-5}-10^{-10}$ \\
Depth of porous medium $h(\mathrm{~m})$ & 0.1 & $1-10^{2}$ \\
Viscosity $\mu$ (Pa s) & $10^{-3}$ & $1-10^{-2}$ \\
Solutal diffusivity $D\left(\mathrm{~m}^{2} \mathrm{~s}^{-1}\right)$ & $10^{-9}$ & $10^{-11}-10^{-13}$ \\
\hline
\end{tabular}




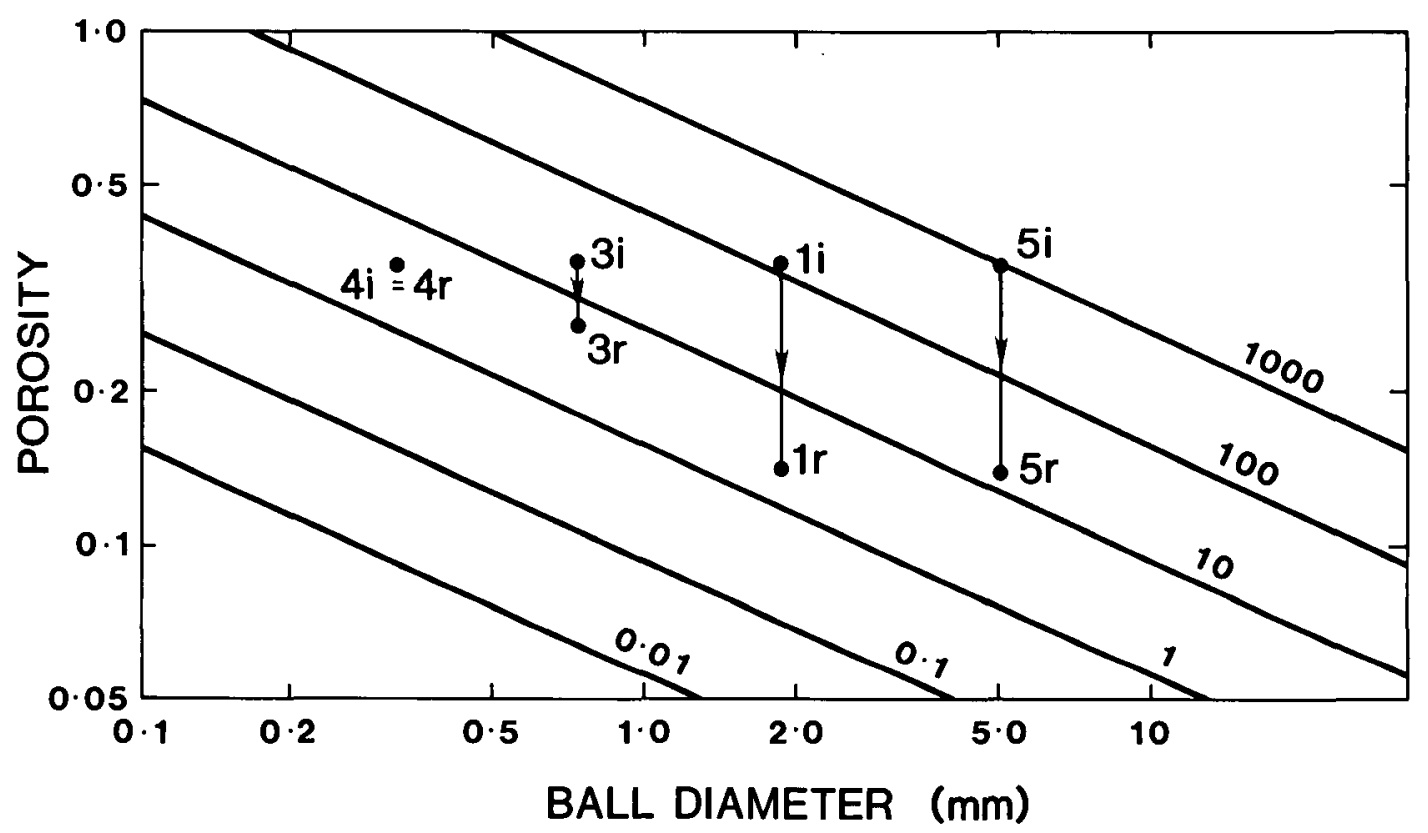

Figure 4. A plot of porosity versus ball diameter for four experiments in the system $\mathrm{CuSO}_{4}-\mathrm{Na}_{2} \mathrm{SO}_{4}-\mathrm{H}_{2} \mathrm{O}$. The numbers 1 , 3, 4 and 5 refer to individual experiments described by Kerr \& Tait (1985a). Each experiment shows the initial porosity (subscript i) and the final residual porosity (subscript $r$ ). The diagram is contoured for the ratio of convective velocity $\left(v_{\mathrm{c}}\right)$ to solidification velocity $\left(v_{\mathrm{s}}\right)$.

convective velocity is greater than the solidification velocity.

For rapidly cooled bodies, such as sills or lava flows solidification velocities are expected to be several metres per year or more. Adcumulus growth would not be expected to be important in such bodies since the solidification velocity exceeds the calculated convection velocities even at large porosities (Figure 5). In medium sized shallow-level intrusions like Rhum, solidification velocities of order of 1 to 0.1 metres per year would be anticipated (Irvine, 1970). Adcumulate growth would be expected within the cumulus piles, but pure adcumulates would not be anticipated by this mechanism. Inspection of Figure 5 indicates that residual porosities of approximately $10 \%$ could be achieved if the convection velocity is $10^{-1}$ metres per year with basaltic magma. Orthocumulates and

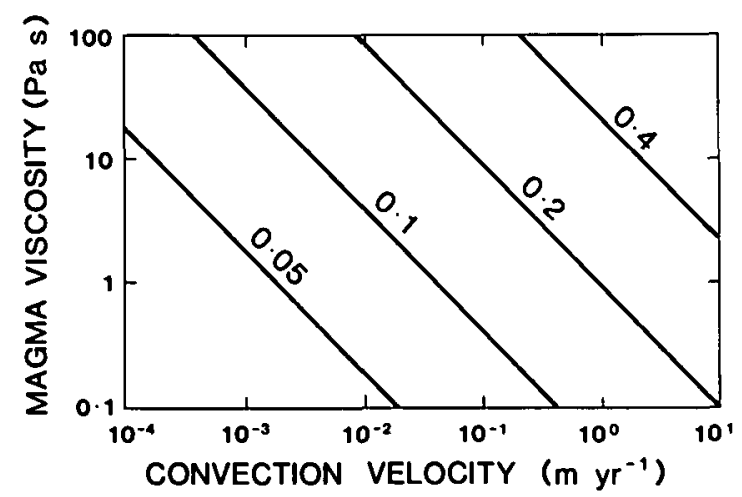

Figure 5. A plot of magma viscosity in Pa s versus convective velocity for different values of porosity. Each line is for a different value of porosity $(\epsilon)$. mesocumulates would be expected to form from cumulate piles with high initial porosity.

In magma chambers that cool very slowly because of their great size or deep crystal level, adcumulus growth within the pile should be most effective. Inspection of Figure 5 suggests that residual porosities of only a few percent could be achieved by this mechanism if the solidification and convective velocities are comparable at values of $10^{-2}$ to $10^{-4} \mathrm{~m}$ per year. As in the 'hardground' model, interstitial adcumulus growth would also be expected to be more prominent in deep and large intrusions.

\section{3.d. Effects of magma chamber replenishment}

The recognition that many magma chambers are open systems, which may be either continuously or periodically replenished by new magma, suggests that this process may also influence postcumulus evolution and consolidation. In particular, replenishment can lead to the situation where primitive high density magma can be emplaced on the floor of the chamber (Huppert \& Sparks, 1980) and can overlie lower density magma within the cumulate pile. This fluid dynamically unstable configuration should lead to the heavy primitive magma sinking into the cumulus pile and lower density residual magma rising and mixing with the overlying magma layer. This kind of process is believed to have occurred in the evolution of the Rhum peridotites and allivalites (Huppert \& Sparks, 1980; Young, 1984; Tait, 1985; Palacz \& Tait, 1985).

This process has been investigated by theoretical and experimental studies (Kerr \& Tait, 1985b). Figure 


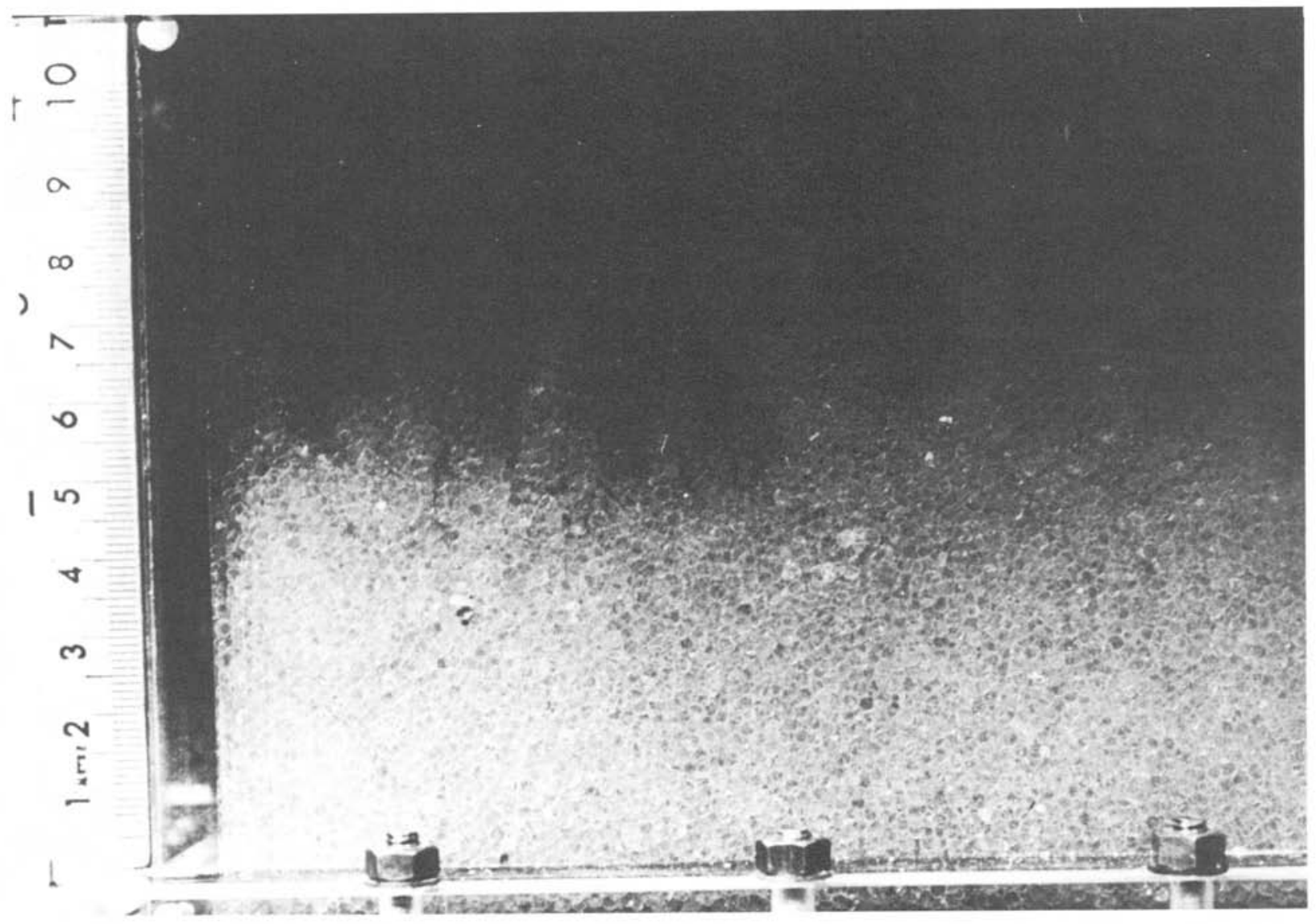

Figure 6. Photograph of an experiment in which a layer of slightly denser fluid is sinking into a porous medium of glass balls. A fingered interface develops. The fluids have equal viscosities (0.1 $\mathrm{Pa}$ s).

6 illustrates a typical experiment where a fluid (usually a glycerine-water mixture) has been emplaced within a porous medium of glass balls until the level of the fluid is just level with the upper surface of the balls. A layer of slightly denser (typically by about $1 \%$ ) dyed fluid is then emplaced. In the photograph the heavier fluid has sunk into the porous medium in a series of finger-like protrusions. The heavy fluid progressively replaces the interstitial light fluid which rises and mixes with the overlying fluid reservoir. Dimensional analysis shows that the velocity of the fingers can be expressed

$$
\nu=\frac{C k g \Delta \rho}{\mu \epsilon},
$$

where C is a constant. Kerr \& Tait $(1985 b)$ have investigated the effects of different viscosities and different initial temperatures on the values of the constant $\mathrm{C}$.

Figure 7 summarizes the application of this work using a typical experimental value of $\mathrm{C}=0.55$, $d=0.2 \mathrm{~cm}$ and $\Delta \rho=5 \times 10^{-2} \mathrm{gm} / \mathrm{cm}^{3}$, which shows how the finger velocity varies with porosity and magma viscosity. Typical calculated velocities range from several metres/year to more than 100 metres/year. These values imply that new magma can penetrate many metres or even hundreds of metres into the underlying cumulate pile, depending strongly on the porosity and to a lesser degree magma viscosities. We note that the velocities are generally higher than either solidification velocities or convective velocities cal-

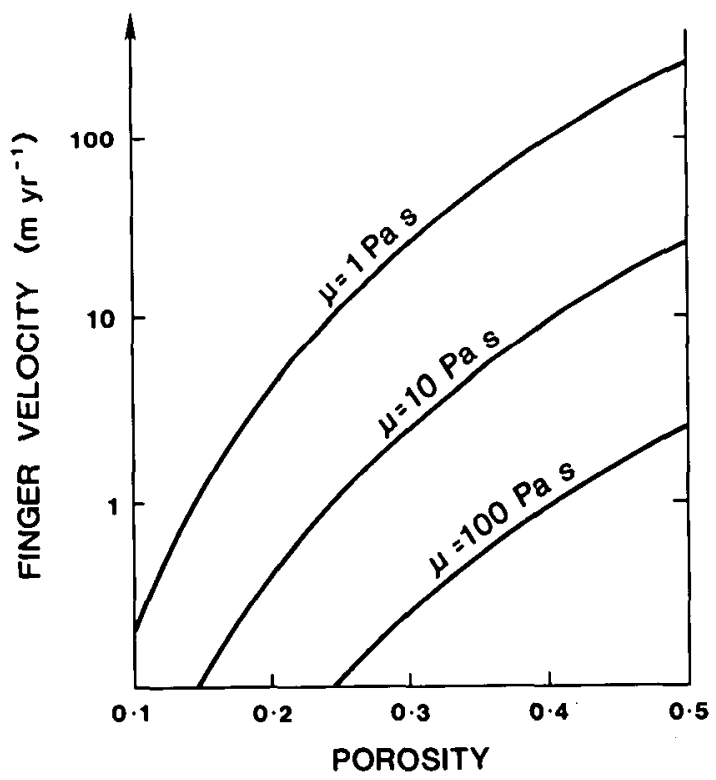

Figure 7. Plot of finger velocity versus porosity for different values of the magma viscosity, calculated from equation (3). In the calculations $\Delta \rho=500 \mathrm{~kg} / \mathrm{m}^{3}, d=0.2 \mathrm{~cm}$ and $\mathrm{C}=\mathbf{0 . 5 5}$. 
culated in Section 3.b, so we infer that this kind of exchange may be important in open system chambers.

In the experiments the glass balls are chemically inert and at the same temperature as the two fluids. In a cumulate pile there are likely to be several complications since the downward moving melt will be out of chemical and thermal equilibrium with the crystalline framework. This situation may lead to precipitation of new minerals, re-equilibration of existing phases (for example, modification of the $\mathrm{Fe} / \mathrm{Mg}$ ratio in olivine and pyroxene) or resorption. Mixing between the two melts could also occur and could lead to precipitation of new phases (Young, 1984). Tait (1985) describes in detail variations of texture, modal abundances, mineral chemistry and isotope ratios in the Rhum peridotites and allivalites of unit 10 that can be attributed to the sinking of denser magma into a cumulus pile with which it is out of chemical equilibrium. Another example of this process appears evident in the Great Dyke in Zimbabwe. Wilson (1982) describes harzburgite layers in cyclic units containing resorbed orthopyroxene cumulus grains and intercumulus olivine. The reaction of opx $\rightarrow \mathrm{ol}+\mathrm{SiO}_{2}$ is apparently possible if the temperature increases. One possibility is that hot primitive magma with olivine on the liquidus sank into an orthopyroxene cumulate pile and resulted in resorption of pyroxene and precipitation of intercumulus olivine.

\section{Compaction}

In explaining the spectrum of rock types from orthocumulates to adcumulates most petrologists have emphasized cementation processes rather than compaction in which the interstitial melt is simply squeezed out by deformation of the crystalline matrix. In principle this process could, for example, form a monomineralic 'adcumulate' rock without any adcumulus growth having taken place. A study of the Muskox intrusion by Irvine (1980) and a theoretical analysis of the compaction of partially molten rock by McKenzie (1984, 1985) and Richter \& McKenzie (1984) have provided arguments for compaction being a major process in layered intrusions.

\section{4.a. Magmatic infiltration metasomatism}

Irvine (1980) observed that the boundaries between major cyclic units in the Muskox intrusion, defined by changes in modal mineralogy, did not coincide with discontinuities in the chemical composition of minerals. Notably the abrupt changes in forsterite content of olivine were displaced several metres above the contact between major olivine pyroxenite and dunite layers, whereas breaks in the nickel contents of olivine coincided with the lithological boundaries. He argued that these relationships were caused by compaction of the cumulate pile and upward flow of differentiated intercumulus melt. This melt would necessarily react with surrounding olivine crystals causing them to become iron-rich. Irvine presented a detailed analysis of the chemical effects of this process and concluded that the abrupt changes in mineral composition would be preserved but displaced upwards with respect to the modal layering.

Richter \& McKenzie (1984) have modelled quantitatively the consequences of compaction of a partially molten layer on the chemistry of the matrix and fluid. They confirm that the kind of displacements observed by Irvine in the Muskox intrusion would indeed be the consequence of compaction.

\section{4.b. Fluid dynamics of compaction}

The theoretical analysis of compaction by McKenzie (1984, 1985) and Richter \& McKenzie (1984) has provided the basis for assessing under what circumstances compaction should be important in layered intrusions. McKenzie (1984) has analysed the compaction of a layer of partially molten rock with constant porosity lying on an impermeable base. For example, this would be an appropriate model for a layer of loosely packed olivine cumulus crystals rapidly formed after a replenishment event. The cause of compaction is that the intercumulus melt has a lower density than the crystalline matrix. Thus there is an imbalance between the lithostatic and hydrostatic pressure gradients. The imbalance results in two-phase flow with compaction of the crystalline matrix and upward flow of the melt. In the case of a compacting layer of constant porosity resting on an impermeable floor McKenzie's analysis demonstrates that compaction will begin at the base and the velocity of escaping melt will increase upwards.

A fundamental parameter emerging from McKenzie's analysis is the compaction length scale, which is the height over which the compaction rate decreases by the factor $e$, and is defined

$$
\delta_{\mathrm{c}}=\left[\frac{\left(\xi+\frac{4}{3} \eta\right)}{\mu} k\right]^{\frac{1}{2}}
$$

where $\xi$ and $\eta$ are the bulk and shear viscosities of the matrix, $\mu$ is the melt viscosity and $k$ the permeability. As McKenzie has discussed in detail, estimation of the matrix properties is uncertain. Richter \& McKenzie (1984) used values of bulk and shear viscosities of $10^{18} \mathrm{~Pa} \mathrm{~s}$. Experimental and theoretical studies (McKenzie, 1985) indicate that this value is appropriate for partially molten mantle. The ratio $k / \mu$ could vary from as much as $10^{-11} \mathrm{~m}^{3} \mathrm{~kg}^{-1} \mathrm{~s}$ in a layer of olivine with $30 \%$ porosity and a low viscosity ( $1 \mathrm{Pas}$ ) picritic liquid to as little as $10^{-16} \mathrm{~m}^{3} \mathrm{~kg}^{-1} \mathrm{~s}$ in a cumulate layer containing high viscosity ( $50 \mathrm{~Pa} \mathrm{~s}$ ) basalt and a porosity of $1 \%$. Thus the compaction length scale might range from as much 
as several kilometres to as little as 0.5 metres depending on the values of the relevant parameters. These length scales could often be much less than the thicknesses of porous cumulate piles in the large magma chambers represented by major layered intrusions, implying that compaction should occur, provided that intercumulus cementation or freezing does not operate on shorter time scales.

For layers of cumulates with a thickness $h$ that is larger than the length scale $\delta_{c}$, the velocity of fluid, $\omega_{0}$, initially emerging out of the top of a layer of initially constant porosity $\epsilon$ can be expressed as

$$
\omega_{0}=\frac{k}{\mu} \frac{(1-\epsilon)}{\epsilon}\left(\rho_{\mathrm{s}}-\rho_{\mathrm{f}}\right) g,
$$

where $\rho_{\mathrm{s}}$ and $\rho_{\mathrm{p}}$ are the densities of the crystalline matrix and intercumulus melt respectively. It should be noted that (S) is strictly only valid at $t=0$ (Appendix B of McKenzie, 1984). However, $\omega_{0}$ serves as a useful measure of the compaction rate, since it can be compared with other characteristic velocities of compositional convection and solidification in a magma chamber.

The velocity of the melt in a partially molten layer of thickness $h$ is zero at the base of the layer and increases upwards. The initial velocity can be calculated from

$$
\omega-\omega=\omega_{0}\left[1-\frac{\cosh \left(\frac{h-z}{\delta_{\mathrm{c}}}\right)}{\cosh \left(\frac{h}{\delta_{\mathrm{c}}}\right)}\right],
$$

where $\omega$ is the melt velocity and $\omega$ the matrix velocity at height $z$ above the base. McKenzie (1985) deduced a characteristic time-scale for compaction:

$$
\tau_{0}=\frac{\delta_{\mathrm{c}}}{\omega_{0}(1-\epsilon)} .
$$

Richter \& McKenzie (1984) have obtained full numerical solutions to the time dependent problem of a compacting layer of constant initial porosity. A particularly useful result concerns the time-scale, $t_{h}$, required to reduce the total amount of fluid in the layer by the factor e. For a layer of thickness $h$ McKenzie (1985) has found that

$$
\frac{t_{h}}{\tau_{0}}=\frac{h}{\delta_{\mathrm{c}}}+\frac{\delta_{\mathrm{c}}}{h} .
$$

The time-scale $t_{h}$ can thus be calculated for the typical layer depths and physical conditions found in layered intrusions and can be compared with the time-scales of other processes such as solidification and convection.

Some general conclusions relevant to the effectiveness of compaction in ultramafic cumulates can be reached by considering the behaviour of olivine cumulate layers of different thicknesses and initial porosities. The formation of such a simple porous layer is thought to be a common phenomenon in the formation of ultramafic cyclic units (Brown, 1956; Huppert \& Sparks, 1980). In the calculations we have assumed a constant viscosity of $3 \mathrm{~Pa} \mathrm{~s}$, a matrix density of $3200 \mathrm{~kg} / \mathrm{m}^{3}$, a melt density of $2700 \mathrm{~kg} / \mathrm{m}^{3}$ and a matrix bulk viscosity $\left(\xi+\frac{4}{3} \eta\right)$ of $10^{18} \mathrm{~Pa}$ s. In calculations of permeability we have followed the arguments of McKenzie (1984) and have used equation 4.4 in that paper and a grain radius of $1 \mathrm{~mm}$. In the following discussion the reader should be aware that there are considerable uncertainties in estimating both the bulk viscosity and the permeability of the matrix at low porosities.

Table 2 lists some characteristic parameters for the compaction of an olivine cumulate layer with intercumulus basaltic melt. For layers of the same thickness as the compaction length scale and the appropriate porosity value (as listed in Table 2) the time-scale required to reduce the amount of intercumulus melt by $\mathrm{e}$ is approximately twice $t_{h}$. Thus significant compaction can occur on time-scales of a few hundred to several thousand years. This result should not differ significantly for other kinds of mafic cumulate rock, since none of the major controlling factors, except porosity, are likely to vary greatly in mafic systems. The characteristic time-scale for the conductive cooling of a sheet of thickness $2 a$ can be usefully compared to these time-scales and is defined as:

$$
t_{\mathrm{c}}=\frac{a^{2}}{\kappa}
$$

where $\kappa$ is the thermal diffusivity $\left(8 \times 10^{7} \mathrm{~m}^{2} \mathrm{~s}^{-1}\right)$. For a $100 \mathrm{~m}$ thick sill $t_{\mathrm{c}}=100$ years, which is a much shorter time-scale than the 4200 years for a compaction length scale of $98 \mathrm{~m}$ (Table 1). Thus compaction would have no influence in such a thin intrusion because it freezes too fast. For a $1 \mathrm{~km}$ thick sill, $t_{\mathrm{c}}=4 \times 10^{4}$ years and is comparable to the time-scales deduced for

Table 2. Characteristic parameters for the compaction of a partially molten layer of olivine and basaltic melt with viscosity of $3 \mathrm{~Pa} s$. Note that for a layer of thickness $\delta_{\mathrm{c}}$ the time-scale, $t_{h}$, is twice $\tau_{0}$.

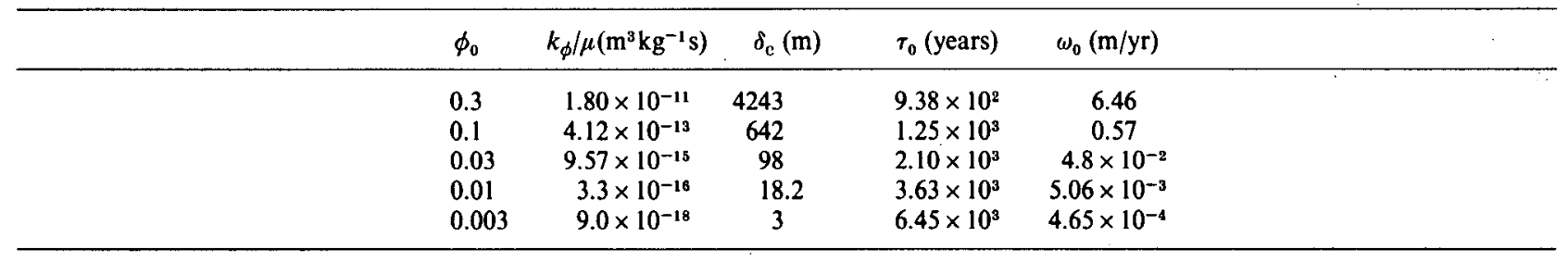




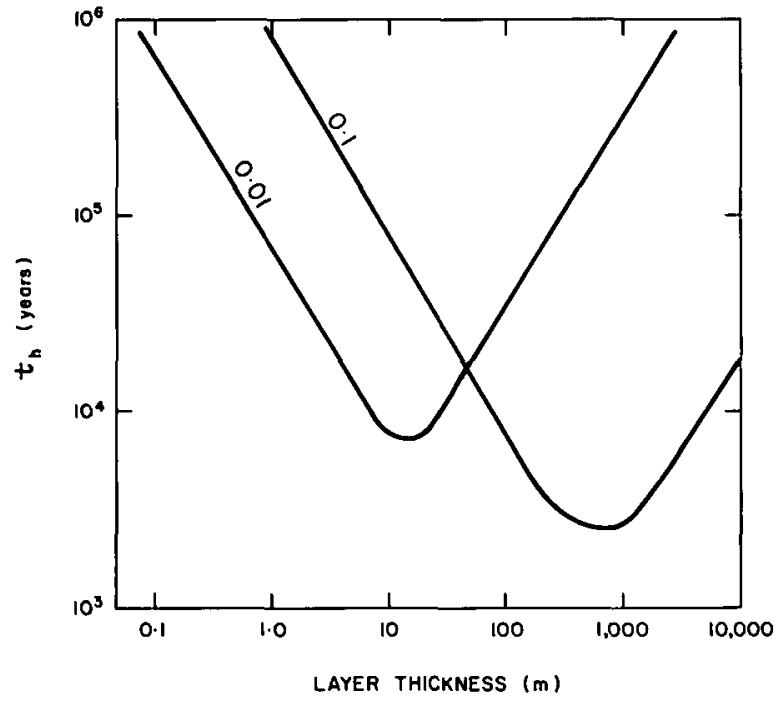

Figure 8. Compaction time scale, $t_{h}$, is plotted against layer thickness for molten layer of olivine and basaltic melt $(\mu=3 \mathrm{~Pa} \mathrm{~s})$. Curves are plotted for two values of porosity, $\epsilon=0.1$ and $\epsilon=0.01$.

values of layers less than $1000 \mathrm{~m}$ thick. Thus compaction might become a factor in intrusions of this dimension. Finally, for a $5 \mathrm{~km}$ thick sill (comparable to the stratigraphic thickness of several large layered intrusions), $t_{\mathrm{c}}=1 \mathrm{~m} . \mathrm{y}$. which is clearly orders of magnitude slower than time-scales deduced for even layers a few metres thick with low porosity. Compaction may therefore be a very effective mechanism of producing adcumulate rocks with low residual porosity in intrusions with dimensions much greater than $1 \mathrm{~km}$. Large slowly cooled intrusions should show the most complete expulsion of intercumulus melt and the most extreme adcumulates.

The numerical calculations of Richter \& McKenzie (1984) yield general results for the time-dependent compaction of partially molten layers. As the thickness of the layer departs increasingly from $\delta_{\mathrm{c}}$, resulting in either $h \gg \delta_{\mathrm{c}}$, or $h \ll \delta_{\mathrm{c}}$, the time scale $t_{h}$ for compaction will increase as is clear from inspecting equation (8). Figure 8 illustrates this effect by plotting the time-scale $t_{h}$ against layer thickness for $\varepsilon=0.1$ and 0.01 . The most rapid removal of intercumulus melt occurs for those layers of thickness $\delta_{\mathrm{c}}$.

Figure 9 shows the variation of the velocity $(\omega-\omega)$ against porosity for different layer thicknesses. Each curve converges onto a single curve that is independent of $h$ and corresponds to the limit $h \gg \delta_{\mathrm{c}}$. The melt velocities can be compared with the solidification velocities which characterize intrusions of different sizes and cooling rates. If the solidification rate is significantly faster than the melt velocity compaction cannot be important because the interstitial melt will be frozen too quickly. In Figure 9 we consider three different solidification rates, respectively appropriate to a $1 \mathrm{~km}$ thick sill, the Rhum intrusion and the Stillwater Complex.

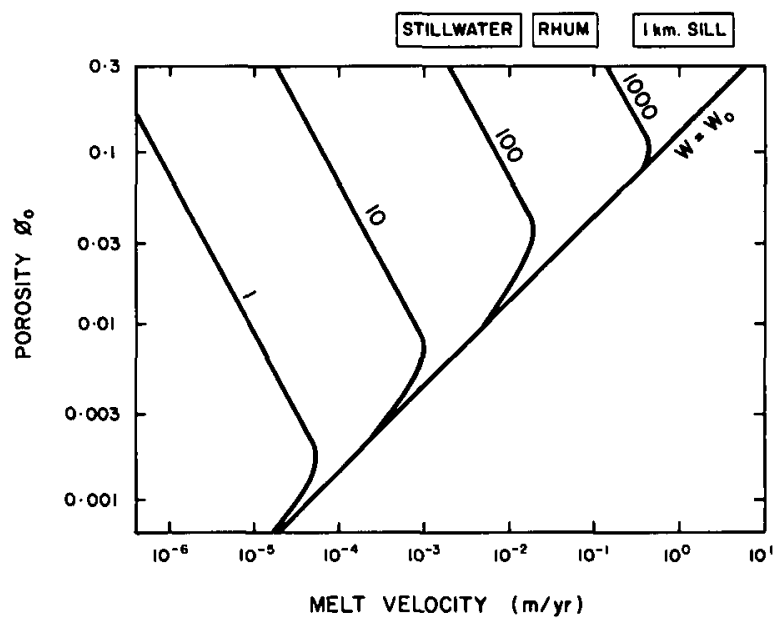

Figure 9. The velocity of liquid emerging from the top of a compacting layer is plotted against porosity for different layer thicknesses in metres. When $\delta_{\mathrm{c}} \leqslant h$ the single line $\omega=\omega_{0}$ is appropriate. Approximate solidification velocities of three intrusions of different dimensions are indicated for comparison.

For a solidification rate of $1 \mathrm{~m} /$ year (as in a $1 \mathrm{~km}$ sill) the melt velocity is always much less. Thus in a layer of cumulate comparable to the sill thickness the compaction rate would be negligible in comparison to freezing rates for a melt viscosity of $3 \mathrm{~Pa}$. In an intrusion of the size of Rhum or Skaergaard a typical rate of solidification might be $10^{-1} \mathrm{~m} /$ year (Irvine, 1970). For cumulate layers greater than $100 \mathrm{~m}$ thick compaction should become a significant factor. For $\mu=3 \mathrm{~Pa} \mathrm{~s}$, the compaction velocity is comparable to or greater than freezing velocities for layers with porosities of a few percent. For slowly cooled plutons such as the Stillwater or Great Dyke, solidification velocities might decrease to as little as $10^{-2} \mathrm{~m} /$ year. Compaction could become a significant factor in layers a few tens of metres thick. Very efficient expulsion of intercumulus melt could be expected with residual porosities of less than $1 \%$. In large intrusions adcumulate rocks are widespread and it appears likely that compaction was an important process. For example the dunite layers of the Great Dyke (Wilson, 1982 ) are almost pure monomineralic rocks ( $>99 \%$ olivine).

\section{4.c. Compaction and cementation}

Cementation and compaction can occur together, but one of these processes may dominate the other in different situations. A quantitative assessment of their relative roles under different boundary conditions is not yet possible, but some qualitative insights can be gained from the experimental and theoretical work reported here.

Both processes may produce adcumulates but both will become insignificant if the solidification rate is high. Adcumulate rocks will therefore form if at least 
one of the characteristic velocities of compaction and convection substantially exceeds the solidification velocity. The following qualitative inferences on their relative importance can be made:

(i) Convection should dominate close to the top of the cumulate pile where the porosity is high and where $h$ is small compared to $\delta_{\mathrm{c}}$.

(ii) Compaction should dominate in thick layers of low porosity $\left(h \gg \delta_{0}\right)$.

(iii) In any given layer in which compositional convection initially dominates at high porosities, compaction will eventually become dominant as the porosity decreases with time.

(iv) In the case of slowly cooled, closed system magma chambers, Morse (1982, personal communication) has argued that extreme adcumulate rocks are generated at or close to the interface with the cumulate pile. This idea has been discussed in section 3.b where little or no interstitial pore space is ever generated. In this situation compaction can have no role and Morse considers that the case for a deep crystal mush has to be made in such intrusions. He argues that compaction is more probable in open systems where accumulation rate may be high. For the case of feldspathic adcumulate in slowly cooled intrusions diagnostic criteria to distinguish extreme surface adcumulus growth from extreme compaction have not been developed. Distinguishing the two models from textural or geological evidence is important and presents an interesting challenge to petrologists.

\section{4.d. Open-system effects}

In open systems like Rhum the accumulation rate may vary by orders of magnitude. For brief periods following a replenishment event, sedimentation rates of olivine from a new layer of primitive magma could be as much as metres or even hundreds of metres per year because of rapid crystallization in response to heat transfer between the liquid layers within the chamber (Huppert \& Sparks, 1980). Then, following such an episode, accumulation rates could return to rates of order $10^{-2} \mathrm{~m} / \mathrm{year}$ due to slow conductive heat loss. Thus wide variations of porosity might develop within the cumulate pile under these circumstances. Scott \& Stevenson (1984) and Richter \& McKenzie (1984) have shown that layers of excess porosity in a partially molten rock can move as waves or pulses with respect to the surrounding matrix and can break up into several zones of high porosity with greater stability. These waves would be expected to interact chemically with their surroundings and could produce replacement features by dissolving out some minerals and reprecipitating others. Geological and geochemical effects that might be ascribed to this process have been described by Irvine (1980) and Butcher, Young \& Faithfull (1985).

\section{Discussion}

This paper reviews some of the ideas on the origin of cumulate rocks, based on fluid dynamical principles. The final chemical and textural characteristics of a rock appear to us to be strongly influenced by two major processes: convective circulation of melt above and within the cumulus pile, and compaction of the crystalline matrix. Both processes involve movement of intercumulus melt and chemical interactions between the melt and matrix. These processes can lead to changes in mineral compositions and textures as the melt re-equilibrates and reacts continually with its matrix. As illustrated by the studies of Irvine (1980), Tait (1985) and Palacz \& Tait (1985) the mineral compositions and isotopic propertiescan be profoundly modified. Adcumulus growth, resorption, reaction textures and replacement textures are likely to prove to be manifestations of intercumulus melt migration and circulation.

The cumulate classification has proved to be useful and resilient to changes in ideas. However, it is now evident that there are several different ways of generating an adcumulate rock. In particular expulsion of melt by compaction is recognized as an important mechanism of generating adcumulate rocks. A challenge for the future will be to recognize diagnostic criteria which will enable the relative importance of cementation and compaction in the evolution of different kinds of cumulate rock to be assessed. Given the richness of fluid dynamical and chemical phenomena that can take place in magma chambers we believe that the terms in cumulate classification can only be used in general descriptive sense. The terms can no longer have more than superficial genetic significance. Interpretation of cumulate rocks will therefore increasingly involve detailed arguments, constrained by the textures, field relations and mineral compositions, that are made with reference to the physical processes that can occur in magma chambers.

Acknowledgements. S.R.T. and R.C.K. acknowledge studentships from N.E.R.C. and Trinity College, Cambridge, respectively. H.H. and R.S.J.S. are supported by the B.P. Venture Research Unit. We acknowledge detailed reviews and comments on an earlier version of the manuscript by Neil Irvine, Tony Morse, Ian Parsons, John Wadsworth and Richard Wilson.

\section{References}

Brown, G. M. 1956. The layered ultrabasic rocks of Rhum, Inner Hebrides. Philosophical Transactions of the Royal Society of London B240, 1-53.

ButCHER, A. R. 1985. Channelled metasomatism in Rhum layered cumulates-evidence from late-stage veins. Geological Magazine 122, 503-18.

Butcher, A. R., Young, I. M. \& Faithfull, J. W. 1985. Finger structures in the Rhum Complex. Geological Magazine 122, 491-502. 
Campbell, I. H. 1978. Some problems with the cumulus theory. Lithos 11, 311-23.

Chen, C. F. \& Turner, J.S. 1980. Crystallization in double-diffusive system. Journal of Geophysical Research 85, 2573-93.

Hess, H. H. 1939. Extreme fractional crystallization of a basaltic magma: the Stillwater igneous complex. Transactions of the American Geophysical Union 3, 431-2.

Hess, H. H. 1960. Stillwater Igneous Complex, Montana, a Quantitative Mineralogical Study. Geological Society of America Memoir no. 80.

Hess, G. B. 1972. Heat and mass transfer-Stillwater Complex. Geological Society of America Bulletin (Harry Hess Volume), Memoir 132, 503-20.

HUPPERT, H. E. \& SPARKs, R. S. J. 1980. The fluid dynamics of a basaltic magma chamber, replenished by an influx of hot, dense ultrabasic magma. Contributions to Mineralogy and Petrology 75, 279-89.

Huppert, H. E. \& Sparks, R. S. J. 1984, Double-diffusive convection due to crystallization in magmas. Annual Reviews of Earth and Planetary Sciences 12, 11-37.

HUPPERT, H. E. \& Worster, M. G. 1985. Dynamic solidification of a binary melt. Nature 314, 703-6.

IRviNE, T. N. 1970. Heat transfer during the solidification of layered intrusions. I. Sheets and sills. Canadian Journal of Earth Sciences 7, 1031-61.

IRVINE, T. N. 1980. Magmatic infiltration metasomatism, double-diffusive fractional crystallization, and adcumulus growth in the Muskox and other layered intrusions. In Physics of Magmatic Processes (ed. R. B. Hargraves), pp. 325-83. Princeton University Press.

IRVINE, T. N. 1982. Terminology for layered intrusions. Journal of Petrology 23, 127-62.

IRvine, T. N., KeITH, D. W. \& TodD, S. G. 1983. The J-M Platinum-Palladium Reef of the Stillwater Complex, Montana. I. Origin by double-diffusive convective magma mixing and implications for the Bushveld Complex. Economic Geology 78, 1287-1334.

JACKSON, E. D. 1961. Primary textures and mineral associations in the ultramafic zone of the Stillwater Complex, Montana. U.S. Geological Survey Professional Paper no. 358, p. 106.

KeRR, R. C. \& TAIT, S. R. (1985a). Crystallization and compositional convection in a porous medium cooled from below with application to layered igneous intrusions. Journal of Geophysical Research (sub judice).

KerR, R. C. \& TAIT, S. R. (1985b). Convective exchange between pore fluid and an overlying reservoir of dense fluid: applications to replenished magma chambers. Earth and Planetary Science Letters (in press).

KerR, R. C. \& TURNER, J. S. 1982. Layered convection and crystal layers in multicomponent systems. Nature 298, 731-3.

LAPWOOD, E. R. 1948. Convection of a fluid in a porous medium. Proceedings of the Cambridge Philosophical Society 44, 508-27.

MCBirney, A. R. 1980. Mixing and unmixing of magmas. Journal of Volcanology and Geothermal Research 7, 357-71.
MCBiRney, A. R. \& Noyes, R. M. 1979. Crystallization and layering of the Skaergaard intrusion. Journal of Petrology 20, 487-554.

MCKenzIE, D. P. 1984. The generation and compaction of partially molten rock. Journal of Petrology 25, 713-65.

MCKenzIE, D. P. 1985. The extraction of magma from the crust and mantle. Earth and Planetary Science Letters (in press)

MORSE, S. A. 1969. Geology of the Kiglapait Layered Intrusion, Labrador. Geological Society of America Memoir no. 112.

MoRSE, S. A. 1979. Kiglapait Geochemistry. II. Petrography Journal of Petrology 20, 591-624.

Morse, S. A. 1982. Adcumulus growth of anorthosite at the base of the Lunar Crust. Journal of Geophysical Research, Supplement to Volumes 87 and 88: Proceedings of the 13th Lunar and Planetary Science Conference, p. 29.

PALACZ, Z. A. \& TAIT, S. R. 1985. Isotopic and geochemical investigation of unit 10 from the Eastern Layered Series of the Rhum Intrusion, Northwest Scotland. Geological Magazine 122, 485-90.

Richter, F. M. \& MCKenzie, D. P. 1984. Dynamical models for melt segregation from a deformable matrix. Journal of Geology 92, 729-40.

Scott, D. R. Stevenson, D. J. 1984. Magma solitons. Geophysical Research Letters 11, 1161-4.

Sparks, R. S. J. \& HupPERT, H. E. 1984. Density changes during the fractional crystallization of basaltic magmas: implications for the evolution of layered intrusions. Contributions to Mineralogy and Petrology 85, 300-9.

TAIT, S. R. 1985. Fluid dynamic and geochemical evolution of cyclic unit 10, Rhum, Eastern Layered Series. Geological Magazine 122, 469-84.

Tait, S. R., Huppert, H. E. \& Sparks, R. S. J. 1984. The role of compositional convection in the formation of adcumulate rocks. Lithos 17, 139-46.

WADSWORTH, W.J. 1985. Terminology of postcumulus processes and products in the Rhum layered intrusion. Geological Magazine 122, 549-54.

WAGER, L. R. \& Brown, G. M. 1968. Layered Igneous Rocks. Edinburgh and London: Oliver \& Boyd.

WAGER, L. R., BROWN, G. M. \& WADSWORTH, W. J. 1960. Types of igneous cumulates. Journal of Petrology 1 , 73-85.

WAGER, L. R.\& DEER, W. A. 1939. Geological investigations in East Greenland. Part III. The petrology of the Skaergaard intrusion, Kangerlwgssauk. Meddelelser om Gronland 105, (4), 1-352.

WiLson, A. H. 1982. The Geology of the Great Dyke of Zimbabwe; The ultramafic rocks. Journal of Petrology 23, 240-92.

WILSON, J. R. \& LARSEN, S. B. 1985. Two-dimensional study of a layered intrusion - the Hyllingen Series, Norway. Geological Magazine 122, 97-124.

Young, I. M. 1984. Mixing of supernatant and interstial fluids in the Rhum layered intrusion. Mineralogical Magazine 48, 345-50.

YounG, I. M. \& Donaldson, C. H. 1985. Formation of granular-textured layers and laminae within the Rhum crystal pile. Geological Magazine 122, 519-28. 